\section{$\underset{\substack{\text { hommes } \\ \text { \& migrations }}}{ }$}

\section{Hommes \& migrations}

Revue française de référence sur les dynamiques

migratoires

$1304 \mid 2013$

Frontières

\title{
D'un pays à l'autre, les exilés de Syrie sont devenus les exclus de Calais
}

\section{Chloé Tisserand}

\section{(2) OpenEdition \\ Journals}

\section{Édition électronique}

URL : http://journals.openedition.org/hommesmigrations/2671

DOI : 10.4000/hommesmigrations.2671

ISSN : 2262-3353

\section{Éditeur}

Musée national de l'histoire de l'immigration

\section{Édition imprimée}

Date de publication : 1 octobre 2013

Pagination : 136-139

ISBN : 978-2-919040-24-7

ISSN : $1142-852 X$

\section{Référence électronique}

Chloé Tisserand, «D'un pays à l'autre, les exilés de Syrie sont devenus les exclus de Calais », Hommes \& migrations [En ligne], 1304 | 2013, mis en ligne le 17 mars 2014, consulté le 22 septembre 2020.

URL : http://journals.openedition.org/hommesmigrations/2671; DOI : https://doi.org/10.4000/ hommesmigrations.2671 


\section{REPÉRAGE}

\section{D'UN PAYS À L'AUTRE, LES EXILÉS DE SYRIE SONT DEVENUS LES EXCLUS DE CALAIS}

CHLOÉ TISSERAND, journaliste à La Voix du Nord de Calais.

$\mathrm{K}$ osovars, Afghans, Kurdes irakiens, Soudanais... Ce sont désormais des Syriens en exode, qui ont rejoint la file d'attente de la distribution des repas à Calais. Ils sont arrivés progressivement depuis l'été 2012 et sont entre 50 et 70 aujourd'hui. La crise syrienne survenue en 2012 et qui a viré au conflit a des répercussions jusque dans la ville des six bourgeois. Le dernier rempart pour ces âmes, habitées par la croyance qu'en Angleterre la vie sera peut-être plus simple. Calais est à la croisée de leurs espérances.

\section{Les nouveaux exilés}

La ville réceptionne depuis dix ans les victimes des conflits, des crises, des dictatures. L'actualité internationale fait écho. "Il y a un effet direct des 'printemps arabes'. En 2011, on a vu à Calais beaucoup de Tunisiens. Lors de la guerre en Libye, on a eu un important flux migratoire en provenance de ce pays, des Africains de l'Est surtout', remarque Philippe Wannesson, président de l'association calaisienne d'aide aux migrants, la Marmite aux idées. "On a aussi beaucoup d'Albanais qui ont été expulsés par l'Angleterre ou qui subissent le contrecoup de la crise économique grecque. Ils n'ont plus de travail et veulent tenter leur chance ailleurs." Des Égyptiens en errance se sont aussi retrouvés, assis sous la pluie, sur le goudron de la cour du lieu de distribution, à manger leurs repas dans des barquettes. Les Syriens sont les derniers arrivés. Difficile à dire s'il y a des "pro-Bachar" et des "anti" à Calais. Ceux rencontrés disent fuir le dictateur et viennent à Calais pour rejoindre l'Angleterre. "Comme par hasard, dans chaque pays traversé et jusqu'en France, terre de transit, l'accueil est à peu près le même : c'est un refus violent ou 'poli"', écrit Smain Laacher. II ajoute: "Le choix de l'Angleterre n'est pas un choix politique fondé sur une analyse comparative des régimes d'hospitalité, français et anglais, en matière de droit d'asile et d'accueil des étrangers." II précise que les migrants ont besoin "d'aller jusqu'au bout", voire le plus loin possible. Ils ne voient pas l'Angleterre comme un eldorado. Ils s'y rendent parce qu'ils sont poussés en avant par la fuite face à la guerre d'abord et ensuite devant la sévérité de la politique d’accueil. À Calais, le phénomène est le même que dans les pays traversés : on fuit des conditions de vie indignes pour tenter de trouver une place meilleure ailleurs.

\section{Expulsions d'un lieu à l'autre}

"François Soulage a interpellé en septembre le président sur la question des migrants car il y a un effet de concentration à Calais, c'est un goulot d'étranglement", rappelle Véronique Devise, présidente de la délégation du Secours catholique du 
Pas-de-Calais. Le président, François Hollande, a répondu à François Soulage en qualifiant la situation des migrants à Calais d'“anormale". II s'est aussi engagé à régulariser 500 Syriens en France. À Calais, les Syriens sont sans cesse expulsés par la police et migrent de squat en squat. Ils sont restés un moment rue Mouron, dans un préfabriqué juxtaposant un grand hangar où s'abritaient des migrants africains. L'endroit, surnommé la "Beer House", est un ancien cash and carry abandonné avec tout son stock de bières. Cet abri a été évacué en septembre par les forces de l'ordre. Philippe Wannesson explique que "les Syriens sont ensuite allés dans l'ancien hôpital désaffecté d'où ils ont été expulsés le lendemain matin. Après, ils ont trouvé un squat rue Duguay-Trouin, d'où ils ont été encore expulsés. Ils se sont alors dispersés en petits groupes. Une partie d'entre eux se sont installés sous l'arcade de l'ancienne douane, jusqu'à ce qu'elle soit obstruée." Il est dans la politique de la municipalité d'être ferme à l'égard des squats. Elle invoque des questions de sécurité pour les migrants qui vivent dans des endroits insalubres et vétustes menaçant de s'écrouler à tout moment. Mais c'est aussi par la peur "d'un nouveau Sangatte" et du fameux "appel d'air" qu'elle justifie sa répression.

\section{La mobilisation des Syriens}

Ces expulsions incessantes ont poussé les Syriens à se mobiliser. "Ils ont adressé un appel au Parlement européen quand il y avait le débat sur les réfugiés syriens, et au Conseil européen lors du débat sur Lampedusa et les réfugiés, mais c'est parce qu'ils ont été sollicités pour ça, ce n'est pas de leur propre initiative", précise Philippe Wannesson. Une soixantaine de Syriens, hommes, femmes et enfants, se sont rendus au port pour bloquer la passerelle piétonne permettant d'accéder aux ferries. Certains menaçaient même de se jeter dans le vide. Le préfet du Pas-de-Calais et le sous-préfet de la ville leur ont proposé de demander l'asile en France. "Aujourd'hui, les Syriens présents ici se sont mis dans une impasse qui ne fera pas évoluer leur situation. Ce que nous pouvons faire, c'est leur donner un statut sur le territoire français, de sorte qu'ils n'aient plus de problèmes", a déclaré le préfet Denis Robin à La Voix du Nord en ajoutant: "Je ne les incite pas à s'installer en France mais à régulariser leur situation en France." Sauf que la majorité des Syriens souhaitent être accueillis en Angleterre.

Une délégation britannique est venue discuter autour de la table des négociations avec un représentant des exilés syriens et des bénévoles lors de la mobilisation. Elle leur a indiqué qu'il n'était pas possible de régulariser tous les Syriens. "Et cette annonce a eu un impact, considère Véronique Devise. Les Syriens ont compris que l'Angleterre n'accueillerait pas plus qu'en France et que la politique d'accueil n'est pas forcément plus facile làbas. Suite à cela, quelques-uns ont demandé l'asile ici, ils étaient six-sept." Ils ont pu déposer leur demande sans avoir de délai d'attente. La plupart des Syriens qui ont manifesté pendant ces deux jours au port ont réussi à passer en Angleterre ou en Suède. "Selon nous, cette action s'est présentée parce qu'une semaine avant, une nuit de veille a été organisée par les associations, raconte Véronique Devise. Cela a permis aux migrants de s'exprimer. L'un d'eux a apparemment eu une prise de parole qui a mobilisé les autres Syriens, c'est ce qui a peut-être préparé leur action au port."

\section{Au nom de meilleures conditions de vie}

Trois jours après, une vingtaine de Syriens se sont posés sur les marches de la mairie avec des pancartes sur lesquelles on pouvait lire: "We need house", "We sleep in the street". Le délégué national de l'opposition syrienne en France a fait son apparition à Calais et a demandé à la mairie des conditions d'accueil décentes. Joe, 27 ans, assis en haut du parvis, explique : "Nous voulons voir 


\section{REPÉRAGE}

le maire de Calais pour lui demander de nous respecter. On dort dans la rue, c'est très dur. On ne veut pas rester en France. Tous, on veut aller en Angleterre. Nous ne voulons pas d'argent mais juste du respect." Ce Syrien, professeur originaire de Damas, est arrivé il y a un mois. Il est d'abord resté au Liban avant de finir par partir : "Ce n'était pas possible de rester en Syrie car tu peux te faire tuer facilement. Et, au Liban, il n'y a pas de travail et tout est sous le contrôle du Hezbollah. Comme nous sommes contre Bachar, ce n'est pas possible de rester." II s'est donc rendu en Égypte : "C'était Morsi à ce moment-là, il était de notre côté. Syriens et Égyptiens étaient égaux. J'avais un travail, je faisais des études, on pouvait avoir tout ce qu'on voulait. Quand il est tombé, tout s'est retourné contre nous." II quitte alors l'Égypte pour la Sicile, où il dit que "c'était très dur", c'est comme ça qu'il s'est retrouvé à Calais. Ahmoud Alhamoud y est depuis deux mois. II vient d'Idleb. "Je ne veux pas demander l'asile ici. J'ai un frère et des amis en Angleterre. Ici, je me sens mal, il n'y a ni douches ni toilettes dans la jungle. Je suis parti en Égypte un an, ensuite en Italie, puis à Calais. Pourquoi ? Parce que la vie s'accomapgne toujours de problèmes politiques que tu ailles dans un endroit ou dans un autre."

\section{Le camp des Syriens}

Si elle a été fortement médiatisée, la mobilisation des Syriens, en termes de conditions d'accueil, n'a pour le moment rien changé. Et les exilés se clochardisent. Ils n'ont rien et dépendent des associations pour survivre. À force d'expulsions, ils ont rejoint un terrain situé près du port de Calais qui se trouve en face du lieu de distribution. Plus d'une trentaine de tentes ont été installées en rang d'oignons. Elles rappellent celles des Petits Frères des Pauvres le long des quais de la Seine. Le rouge a été troqué pour le vert. Dans le camp, visible et à ciel ouvert avec des rues de Calais qui le délimitent, il n'y a que des hommes, des jeunes surtout. "Lors d'un conflit, la famille se déplace dans un premier temps vers les pays voisins, comme au Liban pour les Syriens par exemple. La migration vers l'Europe est secondaire. II y a d'abord une mise à l'abri de la famille et après arrive la décision d'envoyer un de ses membres qui s'installera en Europe et pourra ensuite demander le regroupement familial", décrypte Philippe Wannesson.

Un quadragénaire rondouillard avec un bonnet rouge sur les oreilles reste debout au milieu de toiles kaki qui poussent comme des champignons. Elles sont aussi recouvertes de bâches. "J'ai essayé de passer vingt fois en une semaine en Angleterre mais la police est trop forte, elle me trouve toujours. Une fois, je suis revenu ici à 3 heures du matin et sous la pluie", bougonne-til. Les températures à Calais ont chuté. Le Pakistanais montre les couches de pulls qui recouvrent son corps. II insiste aussi sur le fait qu'il n'a seulement qu'un repas chaud par jour. Comme les autres migrants, il raconte ce même parcours en "sauts de puce": I'Iran, la Turquie, la Grèce, I'Italie, autant de pays où il est resté quelque temps, où il a essayé de trouver un travail et de construire une nouvelle vie. En vain. C'est au bout de cette logique qu'il arrive à Calais. "J'ai quitté le Pakistan parce que jeetais un homme d'affaires là-bas et quand jéchangeais de l'argent, que quelque chose valait 1, et que je le vendais 2, ça c'était du business pour moi. Mais ça c'était haram pour les talibans. Je suis parti à cause d'eux."

Des Syriens passent la tête hors d'une tente. Ils sont cinq ratatinés dans ce mouchoir de poche où les matelas sont des palettes en bois. L'air confiné est humide et pas renouvelé. Ahmed, qui connaît l'anglais, s'avance: "J'étais dans l'armée qui se battait contre le gouvernement, mais 
je l'ai quittée parce qu'on me demandait de tirer sur des femmes et des enfants. Je ne voulais pas tuer des gens de mon pays, alors j'ai fui." II raconte que toute sa famille est en ce moment réfugiée à Beyrouth. II dit qu'il ne veut pas de photo, il ne veut pas que sa mère apprenne sa condition ici. Comme, par exemple, remplir une bouteille d'eau froide, l'emmener dans un endroit caché pour pouvoir se laver. Ou alors les contrôles policiers. "Ils me demandent pourquoi je suis là. Je leur réponds avec évidence qu'on ne sait pas où dormir. On ne peut pas faire autrement", explique Ahmed. Lui ne vit pas dans le camp mais a trouvé un abri dans un garage appartenant aux No Borders. II a rejoint les autres Syriens au camp. Ahmed aime son pays et le Liban. II en parle avec nostalgie et aimerait être là-bas pour retrouver la vie qu'il menait avant la guerre. "Ici, je ne m'habitue pas au froid, je n'ai rien, pas de musique, pas de maison.
J'en vois autour de moi qui deviennent fous", ditil. Un migrant s'approche : il a écrit le nom de la ville sur un bout de papier, C.A.L.A.I.S., et demande quel est le nom de l'endroit où ils vivent. Les tentes, silhouettes de misère, ont été plantées place de l'Europe.

\section{Bibliographie}

Smain Laacher, Après Sangatte. Nouvelles immigrations, nouveaux enjeux, Paris, La Dispute, 2002. Smain Laacher, Le Peuple des clandestins, Paris, Calmann-Lévy, 2007.

Henri Coureau, Ethnologie de la Forme-camp de Sangatte, Paris, Les archives contemporaines, 2007. 